\title{
Dynamic Susceptibility Contrast Perfusion Magnetic Resonance Imaging Demonstrates Reduced Periventricular Cerebral Blood Flow in Dogs with Ventriculomegaly
}

\author{
Martin J. Schmidt ${ }^{*}$, Malgorzata Kolecka', Robert Kirberger ${ }^{2}$ and Antje Hartmann ${ }^{3}$ \\ ${ }^{1}$ Department of Veterinary Clinical Sciences, Clinic for Small Animals, Justus-Liebig-University Giessen, Giessen, Germany, \\ ${ }^{2}$ Companion Animal Clinical Studies, Faculty of Veterinary Science, University of Pretoria, Onderstepoort, South Africa, \\ ${ }^{3}$ Hofheim Veterinary Clinic, Hofheim, Germany
}

OPEN ACCESS

Edited by:

Fintan John McEvoy,

University of Copenhagen,

Denmark

Reviewed by:

Simon Platt,

University of Georgia,

United States

Christopher R. Lamb,

Royal Veterinary College,

United Kingdom

*Correspondence:

Martin J. Schmidt

martin.j.schmidt@vetmed.

uni-giessen.de

Specialty section: This article was submitted

to Veterinary Neurology and Neurosurgery,

a section of the journal

Frontiers in Veterinary Science

Received: 14 June 2017 Accepted: 09 August 2017 Published: 22 August 2017

Citation:

Schmidt MJ, Kolecka M, Kirberger R and Hartmann A (2017) Dynamic Susceptibility Contrast Perfusion Magnetic Resonance Imaging

Demonstrates Reduced

Periventricular Cerebral Blood Flow in Dogs with Ventriculomegaly.

Front. Vet. Sci. 4:137. doi: 10.3389/fvets.2017.00137
The nature of ventriculomegaly in dogs is still a matter of debate. Signs of increased intraventricular pressure and atrophy of the cerebral white matter have been found in dogs with ventriculomegaly, which would imply increased intraventricular pressure and, therefore, a pathological condition, i.e., to some extent. Reduced periventricular blood flow was found in people with high elevated intraventricular pressure. The aim of this study was to compare periventricular brain perfusion in dogs with and without ventriculomegaly using perfusion weighted-magnetic-resonance-imaging to clarify as to whether ventriculomegaly might be associated with an increase in intraventricular pressure. Perfusion was measured in 32 Cavalier King Charles spaniels (CKCS) with ventriculomegaly, $10 \mathrm{CKCSs}$ were examined as a control group. Cerebral blood flow (CBF) was measured using free-hand regions of interest $(\mathrm{ROI})$ in five brain regions: periventricular white matter, caudate nucleus, parietal cortex, hippocampus, and thalamus. CBF was significantly lower in the periventricular white matter of the dogs with ventriculomegaly $(p=0.0029)$ but not in the other ROls. Reduction of periventricular CBF might imply increase of intraventricular pressure in ventriculomegaly.

Keywords: normal pressure hydrocephalus, brachycephaly, magnetic resonance imaging cerebral blood flow, malformation, Cavalier King Charles spaniels

\section{INTRODUCTION}

In brachycephalic dogs, the lateral cerebral ventricles can be relatively large compared to mesaticephalic dogs (1-3). It has been widely accepted that this increase in ventricular volume is not associated with clinical signs and ventriculo-peritoneal shunting is not indicated (4-7). The finding of large lateral cerebral ventricles was referred to as ventriculomegaly in order to differentiate it from clinically relevant internal hydrocephalus (6-9). However, it was shown that signs of increased intraventricular pressure exist in both, dogs with internal hydrocephalus and neurological dysfunction, and also in clinically sound dogs with ventriculomegaly $(9,10)$. Furthermore, a recent morphological study (11) revealed that larger cerebral ventricles in brachycephalic dogs are associated with white matter loss as occurs in conventional internal hydrocephalus $(12,13)$. Based on these changes, it must be considered that canine ventriculomegaly is not a physiological variant of ventricular dimensions as previously reported $(1-3,5-7)$ but may be a preliminary or arrested form of internal hydrocephalus. 
Many forms of hydrocephalus in humans can be associated with a reduction in periventricular cerebral blood flow (CBF) (14-16). CBF refers to the volume of blood per unit time passing through a given region of brain tissue, commonly measured in milliliter per minute per $100 \mathrm{~g}$ of brain tissue. This was also shown in dogs with experimentally induced internal hydrocephalus (17). The impaired perfusion is believed to be caused by increased cerebrospinal fluid (CSF) pressure and ventricular distension, which causes stretching of periventricular blood vessels and white matter fibers and consequently functional, and later to structural damage of periventricular white matter $(13,16,18)$. The reversibility of hydrocephalus symptoms was associated with improved $\mathrm{CBF}$ in the periventricular white matter after shunting, which supports this hypothesis (19).

Magnetic resonance imaging (MRI) can be used to non-invasively measure CBF in humans and in dogs (20-23). Dynamic susceptibility contrast perfusion MRI (DSC-PMRI) allow to quantify blood volume, which passes through the vascular system of brain tissue. The technique quantifies the induced signal loss caused by paramagnetic contrast agents, which is proportional to the amount of blood in the microvasculature (24-26). DSC-PMRI was used demonstrate reduced regional $\mathrm{CBF}$ in the periventricular white matter in human patients with hydrocephalus $(27,28)$.

We hypothesize that periventricular CBF might also be decreased in dogs with ventriculomegaly just as in human patients with hydrocephalus. To investigate this hypothesis, we aim to measure brain perfusion using DSC-PMRI in dogs with ventriculomegaly and compare it to brain perfusion in normal dogs.

\section{MATERIALS AND METHODS}

\section{Study Population}

Cavalier King Charles spaniels (CKCS) were chosen for the study. From dogs that underwent MRI-scanning of the head and cervical spine for breeding selection against syringomyelia (SM), CKCSs with ventriculomegaly were selected for the study group. The presence of ventriculomegaly was based on the following criteria. Dogs with a normal ventricular system have very narrow and slit-like horns of the lateral ventricles. In the finding of ventriculomegaly, the interpreter subjectively noted a greater proportion of the intracranial volume occupied by the lateral ventricles. The closely spaced walls of the temporal horns and/ or the olfactory recesses were separated by cerebrospinal fluid (Figures 1C,D: yellow arrows) in these brains and the lacking of a septum pellucidum created a large connection between the first and second ventricles (Figures 1C,D: blue arrows). CKCSs without these findings were used as a control (Figures 1A,B). The groups were age and weight matched. Before scanning, each dog underwent a general and neurological examination by a

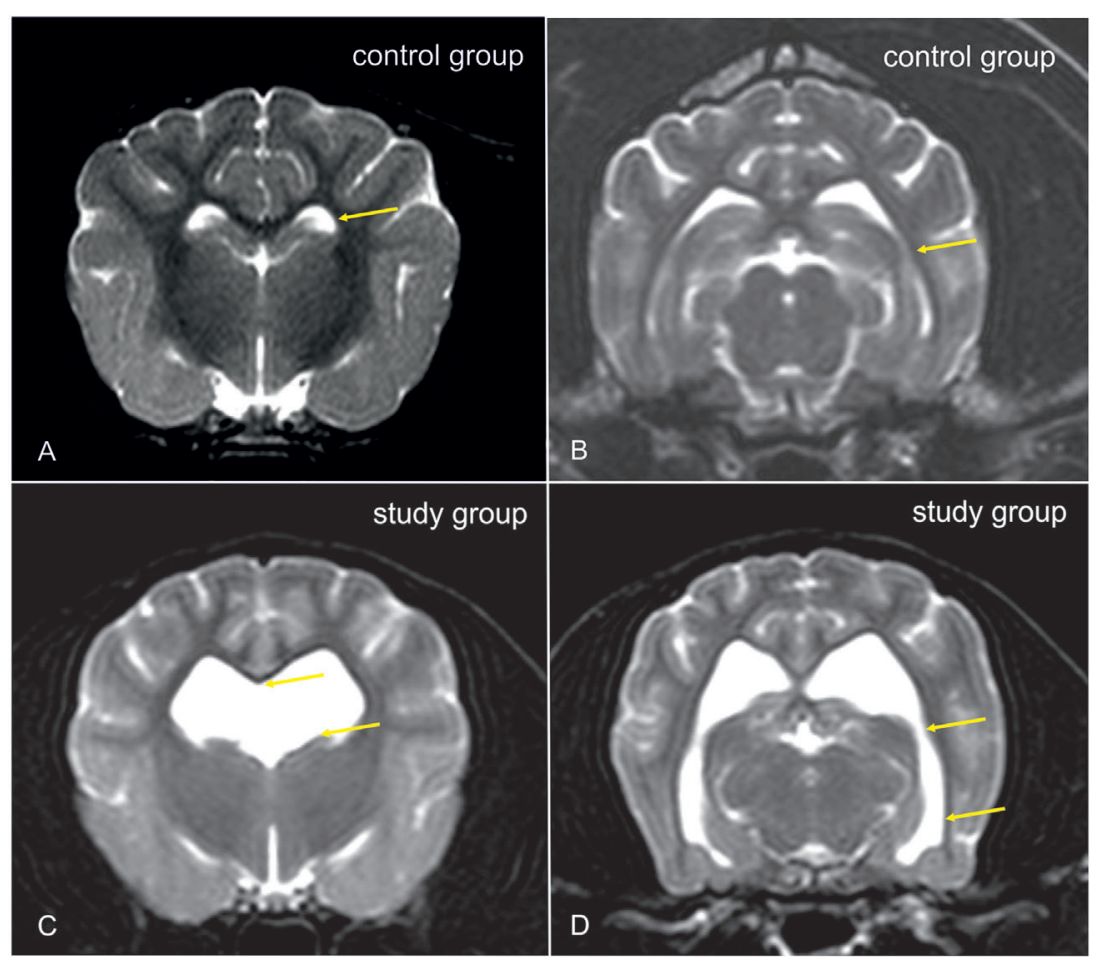

FIGURE 1 | Transverse T2-weighted images of the brain of a Cavalier King Charles spaniel (CKCS) with normal lateral cerebral ventricles [control group, (A,B)] and a CKCS with ventriculomegaly [study group, (C,D)]. In the study group, a greater proportion of the brain volume is occupied by the lateral ventricles. The closely spaced walls of the temporal horns [(B,D): yellow arrows] and/or the olfactory recesses were separated by cerebrospinal fluid (CSF) in these brains, and the lacking of a septum pellucidum created a large connection between the first and second ventricles [(C): yellow arrows]. 
board-certified neurologist. Dogs with SM or neurological signs were not included into the study. All dogs were positive for the morphological changes consistent with Chiari-like malformation. Complete blood count, biochemistry panel, and electrolytes were determined before examination.

\section{Ethics Statement}

All investigations were conducted in strict compliance with the restrictions of the German Animal Protection Law. All dogs from the study and control group were client owned and lived with their owners. The owners of the dogs gave permission for their animals to be used in this investigation. The study was approved by the Committee on the Ethics of Animal Experiments of the Justus-Liebig-University, Giessen.

\section{Anesthesia Protocol}

Standard intravenous catheters (18 gage) were placed in the right or left cephalic vein. Diazepam $(0.5 \mathrm{mg} / \mathrm{kg})$ was administered IV, and anesthesia was induced with propofol (2-4 mg/kg, IV). Dogs were endotracheally intubated and anesthesia was maintained with $1.5-2 \%$ isoflurane in oxygen. Dogs were automatically ventilated throughout the MRI examination and kept normocapnic (35-45 mmHg). $\mathrm{CO}_{2}$ was measured using side stream capnography from the endotracheal tube.

\section{Imaging Technique Standard Head Examination}

In order to diagnose structural changes consistent with Chiari-like malformation and SM, a standard MRI examination of the brain and spinal cord was performed prior to the perfusion studies. Imaging was performed using a 1 T MRI scanner (Gyroscan Intera, Phillips, Hamburg, Germany) and a two-part surface coil consisting of two elliptical elements, which were placed on the right and left sides of the head. Dogs were examined in sternal recumbency with their neck in extension sagittal, dorsal, and transverse images were obtained using T2-Turbospin echo sequences (TE: $120 \mathrm{~ms}$, TR: 2,900 ms). Transverse FLAIR images and dorsal T1-weighted gradient echo images were acquired before and after contrast (i.e., after perfusion study) medium administration to exclude structural brain abnormalities. Field of view was $180 \mathrm{~mm} \times 180 \mathrm{~mm}$, matrix was $288 \times 288$. Slice thickness varied from 2 to $3 \mathrm{~mm}$. The cervical spine was examined until the first the first thoracic vertebra. Sagittal T2-weighted images were obtained. If the presence of SM was confirmed, transverse gradient-echo images were obtained over the whole extension of the SM.

\section{Perfusion-Weighted Imaging}

Perfusion-weighted images were acquired by use of a dynamic multishot fast-field echo-echo-planar imaging sequence. Slice orientation was parallel to the base of the skull. In total, 40 dynamics/slices were acquired in a dorsal plane with a time gap of $1.6 \mathrm{~ms}$. At the $10 \mathrm{th}$ dynamic, $0.2 \mathrm{mmol} / \mathrm{kg}$ gadoteric acid was injected at a rate of $5 \mathrm{ml} / \mathrm{s}$ using a double-headed injection pump (Accutron MR, Medtron, Saarbrücken, Germany). The injection of contrast medium was followed by a $20-\mathrm{ml}$ injection of isotonic Ringer solution.

\section{Perfusion Analysis}

Image analysis was performed using a commercially available software (Stroketool, Digital Image Solutions, Frechen, Germany). The program works with established perfusion calculation algorithms (SVD-deconvolution) for quantitative perfusion imaging, which takes the arterial input function (AIF) into account (29, 30). The AIF was determined at the level of the middle cerebral artery. In this model, CBF measurements are based on time course signal changes after the infusion of a bolus of gadolinium in each voxel of the brain tissue in comparison of the same changes in the middle cerebral artery.

Regions of interest (ROIs) were manually drawn around the periventricular white matter of the frontal horn (rostral limb of the internal capsule), the caudate nucleus, the thalamus, and the cerebral cortex adjacent to the internal capsule in both hemispheres by one investigator. These ROIs were delineated on corresponding dorsal T2-weighted images linked with perfusion weighted images. CBF was measured and compared between groups as these are the most important perfusion parameters related to cerebral hemodynamic changes in human hydrocephalus $(15,31-33)$. The software calculated the CBF as the blood volume in the brain in a given period of time measured in $\mathrm{ml} / 100 \mathrm{~g} / \mathrm{min}$. The software creates color-coded maps for the estimated parameters from gray scale MR images. In these CBF-maps, red are being highly perfused areas, dark blue indicating extremely low, and green being intermediate perfusion.

\section{Statistical Analysis}

All statistical analyses were performed by use of commercially available software (BMDP Statistical Software, Inc., Los Angeles, CA, USA). The Shapiro-Wilk test was used to assess for normality in datasets. Means and SDs were calculated for normally distributed data. The values for each region were computed as the average values of both sides. Mean values for CBF were compared by use of two-tailed independent sample $t$-tests. The level of significance was set to 0.01 for all tests. To test homogeneity of the groups, the relative frequency of male and female animals in the groups was compared using Fisher's exact test.

\section{RESULTS}

\section{Animals}

Group 1 (normal CKCSs) comprised four male and six female dogs weighing $6-9 \mathrm{~kg}$ (mean $7.53 \pm 1.19$ ). Group 2 (CKCSs with ventriculomegaly) included 13 male and 19 female dogs weighing 6-9 kg (mean $7.54 \pm 1.05$ ). Fisher exact test revealed no statistically difference between the number of females and males between groups $(p=1)$.

\section{Perfusion Measurement}

Mean CBF values are summarized in Table 1. Significantly lower $\mathrm{CBF}$ was found in the periventricular white matter of dogs with ventriculomegaly (Figure 2). Values computed from other ROIs were not significantly different between groups. 
TABLE 1 | Mean \pm SD quantitative estimates of CBF for the regions of interest evaluated in normal Cavalier King Charles spaniels and dogs with ventriculomegaly.

\begin{tabular}{|c|c|c|c|c|}
\hline & Periventricular white matter & Caudate nucleus & Thalamus & Cerebral cortex \\
\hline CBF normal dogs & $100.5 \pm 5.3$ & $101.2 \pm 9.97$ & $94.44 \pm 21.56$ & $102.20 \pm 4.3$ \\
\hline CBF dogs with ventriculomegaly & $42.05 \pm 10.88$ & $85.55 \pm 21.33$ & $50.56 \pm 28.66$ & $97.53 \pm 6.3$ \\
\hline$p$-Value & 0.0029 & 0.077 & 0.367 & 0.0527 \\
\hline
\end{tabular}

CBF, cerebral blood flow (milliliter per minute per $100 \mathrm{~g}$ of brain tissue).

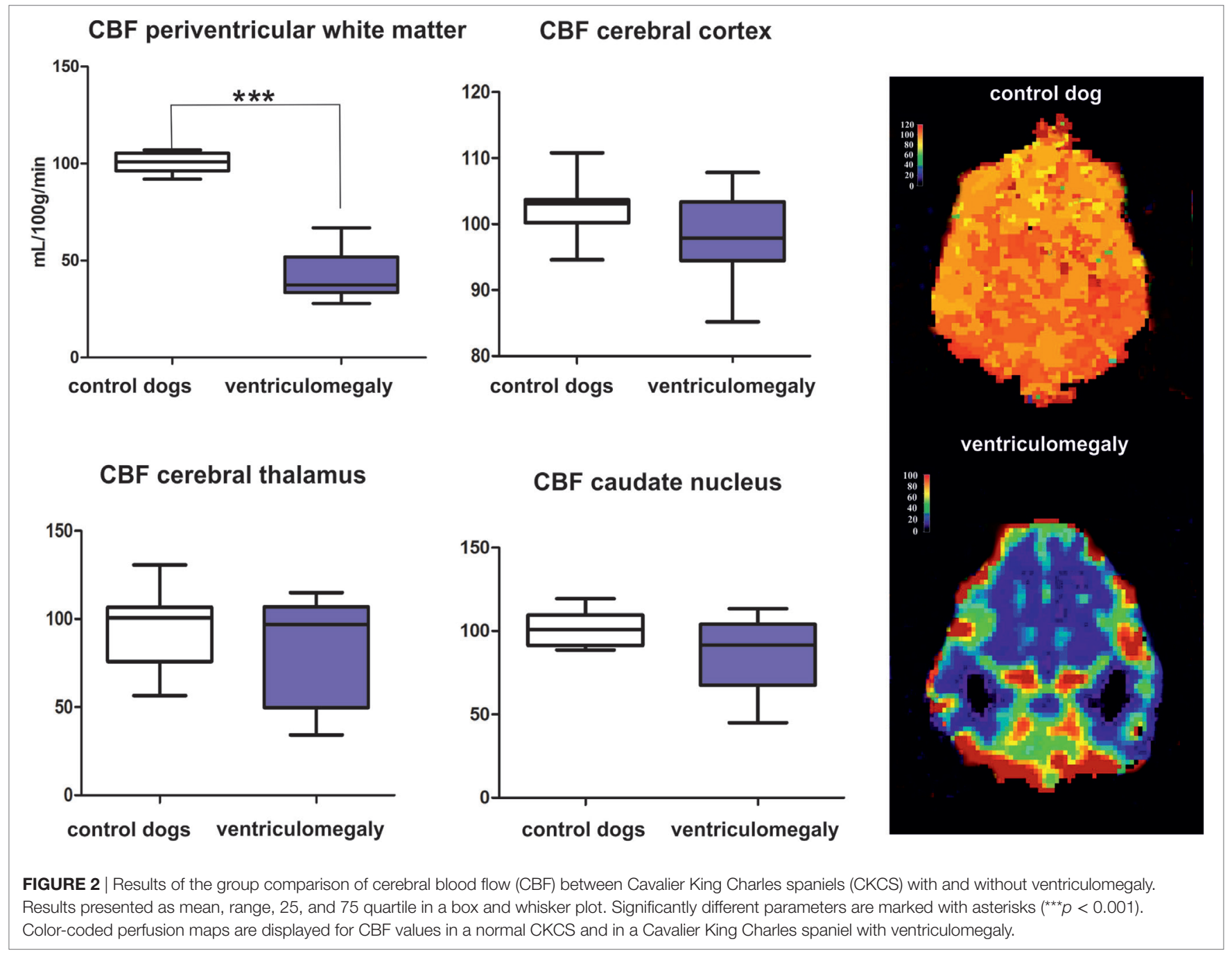

\section{DISCUSSION}

The nature and origin of ventriculomegaly in dogs remain controversial and not well elucidated $(11,34,35)$. Signs of increased intraventricular pressure (elevated corpus callosum, a distended third ventricle and a deformed interthalamic adhesion as well as dilated olfactory recesses) (9) and atrophy of the cerebral white matter (11) have been found in dogs with ventriculomegaly, which would imply a pathological condition to some extent. Here, we examined the regional CBF in CKCSs with ventriculomegaly compared to normal controls using DSC-PMRI with a gadolinium-based contrast agent. The results of the investigation indicate that $\mathrm{CBF}$ is reduced in the periventricular white matter of CKCSs with ventriculomegaly.

MR-perfusion techniques have proven to be feasible in dogs $(22,23)$ but accuracy and validity of the method for its standard use must be further evaluated. Positron emission tomography (PET) is considered to be the gold standard for studying cerebral hemodynamics (36). However, a PET and perfusion-based MR techniques of CBF measurements were found to be highly consistent in studies involving both humans $(24,37,38)$ and dogs $(39,40)$. The values extracted from our study of clinically normal CKCSs come close to those measured in studies using MRI- or CT-based perfusion studies $(39,40)$, but differ from 
others $(17,22)$. However, several factors limit the comparison of the present investigation with other studies. The technique is sensitive to physiological variations, which reduce measurement reproducibility. A source of potential error could originate from the use of different anesthetic drugs that can have a significant effect on cerebral blood vessels. Volatile anesthetics can cause cerebral vasodilatation and thereby an increase in CBF (41-43). Spatial resolution disparities and low signal-to-noise ratios in the perfusion-weighted images make the precise location of the ROIs difficult. The choice of arterial input can have an effect on measuring $\mathrm{CBF}$ (44). Missing standardization of acquisition parameters and use of variable postprocessing software constitutes another challenge for the comparison of data acquired in different studies (45). In addition, this study differs in that we have used combined and averaged measurements from the left and right forebrain in order to discount for known differences between the hemispheres (22).

The findings of ventricular enlargement that correlates with white matter atrophy as well as the reduction in periventricular $\mathrm{CBF}$ found here are comparable to findings in human normal pressure hydrocephalus $(\mathrm{NPH})$ that predominantly affects the elderly $(11,46,47)$. Visible obstructions in the ventricular system cannot be detected in both, humans with NPH and dogs with ventriculomegaly. Studies in elderly people have shown decreased CBF in the periventricular white matter in NPH patients that gradually normalized toward the subcortical white matter (48). This inadequate blood supply eventually may produce structural white matter damage and gradual atrophy (49). However, reduced CBF is also found in the frontal cerebral cortex, thalamus, and in the basal nuclei in patients with NPH (31, 32, 48, 50-52). Although we could not find significant differences between the dogs in these brain areas, the SD of averaged $\mathrm{CBF}$ in the dogs with ventriculomegaly was relatively high for these ROIs with some of the patients also having low measurements in the caudate nucleus and the thalamus (Figure 1). CBF changes in other tissue areas can, therefore, not be totally ruled out.

Based on data on this and other studies, it seems plausible that ventriculomegaly may be a preliminary stage or an arrested form of internal hydrocephalus. Ventriculomegaly has been attributed to disturbances in CSF dynamics in dogs by some authors (11, $34,35)$ that may be caused by osseous obstruction of the foramen magnum, reducing the amounts of CSF expelled from the cranial cavity. Craniocervical junction anomalies that reduce the CSF outflow tract of toward the cisterna magna are often found in small brachycephalic dogs (53). The presence of a widened mesencephalic aqueduct and aqueductal CSF flow void may support the hypothesis of a hyperdynamic CSF flow through the aqueduct as a consequence of an impaired CSF outflow from the skull $(9,10,54)$.

Scrivani introduced to model of a reduced intracranial compliance for dogs with disturbed CSF hemodynamics and ventricular distension (54). Following systolic expansion of the intracranial arteries, the following increase of intracranial pressure is balanced by expulsion of venous blood and CSF from the cranial cavity. If intracranial compliance is reduced, a higher CSF volume is forced from the lateral ventricles through the aqueduct with a higher velocity. The resulting signal void sign in the aqueduct was proposed to be a marker of reduced intracranial compliance in small brachycephalic dogs (54). Compared to mesaticephalic dogs, the cranial capacity, i.e., the part of the cranial cavity that is not occupied by brain tissue, is reduced in brachycephalic dogs (55), which may impair brain expansion during systole. The jugular foramen can be small in the brachycephalic CKCS (56), which may also reduce venting of venous blood from the cranial cavity. Both effects may contribute to a reduced intracranial compliance.

The influence of ventriculomegaly on brain function in dogs is unclear $(4,8,57)$. Human patients with NPH show characteristic triad of neural function deficits including gait impairment, dementia, and urinary incontinence $(58,59)$. This could not be documented in dogs using conventional clinical evaluation. Detailed behavioral studies and breed-specific gait analyses are necessary to clarify the impact of ventriculomegaly on brain function and whether ventriculomegaly might be an indication for CSF shunting procedures in dogs.

\section{CONCLUSION}

Cerebral blood flow can be reduced in periventricular white matter in CKCSs with ventriculomegaly, which makes some increase of intraventricular pressure likely.

\section{ETHICS STATEMENT}

The study was approved by the ethics committee of the Veterinary Faculty of the Justus Liebig University.

\section{AUTHOR CONTRIBUTIONS}

Conceived and designed the experiments: $\mathrm{AH}$ and MS. Performed the experiments: AH, MK, and MS. Analyzed the data: MS. Contributed reagents/materials/analysis tools: AH and MS. Wrote the paper: AH, MS, and RK.

\section{REFERENCES}

1. Kii S, Uzuka Y, Taura Y, Nakaichi M, Takeuchi A, Inokuma H, et al. Magnetic resonance imaging of the lateral ventricles in beagle-type dogs. Vet Radiol Ultrasound (1997) 38:430-3. doi:10.1111/j.1740-8261.1997.tb00866.x

2. De Haan CE, Kraft SL, Gavin PR, Wendling LR, Griebenow ML. Normal variation in size of the lateral ventricles of the Labrador Retriever dog as assessed by magnetic resonance imaging. Vet Radiol Ultrasound (1994) 35:83-6. doi:10.11 11/j.1740-8261.1994.tb00191.x

3. Vullo T, Korenman E, Manzo RP, Gomez DG, Deck MD, Cahill PT. Diagnosis of cerebral ventriculomegaly in normal adult beagles using quantitative MRI. Vet Radiol Ultrasound (1997) 38:277-81. doi:10.1111/j.1740-8261.1997. tb00855.x

4. Driver CJ, Chandler K, Walmsley G, Shihab N, Volk HA. The association between Chiari-like malformation, ventriculomegaly and seizures in cavalier King Charles spaniels. Vet J (2013) 195:235-7. doi:10.1016/j.tvjl.2012.05.014

5. Schwarz T, Saunders J. Veterinary Computed Tomography. Iowa, USA: Wiley Blackwell (2012). 185 p. 
6. Gavin PR, Bagley RS. Practical Small Animal MRI. Iowa, USA: Wiley Blackwell (2011). $64 \mathrm{p}$.

7. Thomas B. Hydrocephalus in dogs and cats. Vet Clin North Am Small Anim Pract (2010) 40:143-59. doi:10.1016/j.cvsm.2009.09.008

8. Saito M, Olby NJ, Spaulding K, Muñana K, Sharp NJ. Relationship among basilar artery resistance index, degree of ventriculomegaly, and clinical signs in hydrocephalic dogs. Vet Radiol Ultrasound (2003) 44:687-94. doi:10.111 1/j.1740-8261.2003.tb00532.x

9. Laubner S, Ondreka N, Failing K, Kramer M, Schmidt MJ. Magnetic resonance imaging signs of high intraventricular pressure - comparison of findings in dogs with clinically relevant internal hydrocephalus and asymptomatic dogs with ventriculomegaly. BMC Vet Res (2015) 11:181. doi:10.1186/ s12917-015-0479-5

10. Ryan CT, Glass EN, Seiler G, Zwingenberger AL, Mai W. Magnetic resonance imaging findings associated with lateral cerebral ventriculomegaly in English Bulldogs. Vet Radiol Ultrasound (2014) 55:292-9. doi:10.1111/vru.12123

11. Schmidt MJ, Laubner S, Kolecka M, Failing K, Moritz A, Kramer M, et al. Comparison of the relationship between cerebral white matter and grey matter in normal dogs and dogs with lateral ventricular enlargement. PLoS One (2015) 10:e0124174. doi:10.1371/journal.pone.0124174

12. Wünschmann A, Oglesbee M. Periventricular changes associated with spontaneous canine hydrocephalus. Vet Pathol (2001) 38:67-73. doi:10.1354/ vp.38-1-67

13. Del Bigio MR. Neuropathological changes caused by hydrocephalus. Acta Neuropathol (1993) 85:573-85. doi:10.1007/BF00334666

14. Mataró M, Poca MA, Salgado-Pineda P, Castell-Conesa J, Sahuquillo J, DíezCastro MJ, et al. Postsurgical cerebral perfusion changes in idiopathic normal pressure hydrocephalus: a statistical parametric mapping study of SPECT images. J Nucl Med (2003) 44:1884-9.

15. Mori K, Maeda M, Asegawa S, Iwata J. Quantitative local cerebral blood flow change after cerebrospinal fluid removal in patients with normal pressure hydrocephalus measured by a double injection method with $\mathrm{N}$-isopropyl-p[123I] iodoamphetamine. Acta Neurochir (2002) 144:255-63. doi:10.1007/ s007010200033

16. Nakada J, Oka N, Nagahori T, Endo S, Takaku A. Changes in the cerebral vascular bed in experimental hydrocephalus: an angio-architectural and histological study. Acta Neurochir (Wien) (1992) 114:43-50. doi:10.1007/ BF01401113

17. Dombrowski SM, Schenk S, Leichliter A, Leibson Z, Fukamachi K, Luciano MG. Chronic hydrocephalus-induced changes in cerebral blood flow: mediation through cardiac effects. J Cereb Blood Flow Metab (2006) 26:1298-310. doi:10.1038/sj.jcbfm.9600282

18. Goh D, Minns RA. Intracranial pressure and cerebral arterial flow velocity indices in childhood hydrocephalus: current review. Child Nerv Syst (1995) 11:392-6. doi:10.1007/BF00717403

19. Klinge PM, Brooks DJ, Samii A, Weckesser E, van den Hoff J, Fricke H, et al. Correlates of local cerebral blood flow (CBF) in normal pressure hydrocephalus patients before and after shunting - a retrospective analysis of $[(15) \mathrm{O}] \mathrm{H}(2)$ O PET-CBF studies in 65 patients. Clin Neurol Neurosurg (2008) 110:369-75. doi:10.1016/j.clineuro.2007.12.019

20. Petrella JR, Provenzale JM. MR perfusion imaging of the brain: techniques and applications. Am J Roentgenol (2000) 175:207-19. doi:10.2214/ajr.175.1. 1750207

21. Tidwell AS, Robertson ID. Magnetic resonance imaging of normal and abnormal brain perfusion. Vet Radiol Ultrasound (2011) 52:62-71. doi:10.1111/j.1740-8261.2010.01786.x

22. Hartmann A, Driesen A, Lautenschläger IE, Scholz VB, Schmidt MJ. Quantitative analysis of brain perfusion in healthy dogs by means of magnetic resonance imaging. Am J Vet Res (2016) 77:1227-35. doi:10.2460/ajvr. 77.11 .1227

23. Stadler KL, Pease AP, Ballegeer A. Dynamic susceptibility contrast magnetic resonance imaging protocol of the normal canine brain. Front Vet Sci (2017) 4:41. doi:10.3389/fvets.2017.00041

24. Østergaard L, Johannsen P, Høst-Poulsen P, Gyldensted C, Rosen BR. Cerebral blood flow measurements by magnetic resonance imaging bolus tracking: comparison with $[15 \mathrm{O}] \mathrm{H} 2 \mathrm{O}$ positron emission tomography in humans. J Cereb Blood Flow Metab (1998) 18:935-40. doi:10.1097/00004647199809000-00002
25. Giesel FL, Wüstenberg T, Bongers A, Weber M, Zechmann C, Weber MA, et al. MR-based methods of the functional imaging of the central nervous system. Röfo (2005) 177:714-30.

26. Barbier EL, Lamalle L, Décorps M. Methodology of brain perfusion imaging. J Magn Reson Imaging (2001) 13:496-520. doi:10.1002/jmri.1073

27. Tanaka A, Kimura M, Nakayama Y, Yoshinaga S, Tomonaga M. Cerebral blood flow and autoregulation in normal pressure hydrocephalus. Neurosurgery (1997) 40:1161-7. doi:10.1097/00006123-199706000-00009

28. Walter C, Hertel F, Naumann E, Mörsdorf M. Alteration of cerebral perfusion in patients with idiopathic normal pressure hydrocephalus measured by $3 \mathrm{D}$ perfusion weighted magnetic resonance imaging. J Neurol (2005) 252:1465-71. doi:10.1007/s00415-005-0891-z

29. Østergaard L, Weisskoff RM, Chesler DA. High resolution measurement of cerebral blood flow using intravascular tracer bolus passages. Part I: mathematical approach and statistical analysis. Magn Reson Med (1996) 36:715-25. doi:10.1002/mrm.1910360510

30. Østergaard L, Sorensen AG, Kwong KK, Weisskoff RM, Gyldensted C, Rosen BR. High resolution measurement of cerebral blood flow using intravascular tracer bolus passages. Part II: experimental comparison and preliminary results. Magn Reson Med (1996) 36:726-36. doi:10.1002/ mrm. 1910360511

31. Ziegelitz D, Arvidsson J, Hellström P, Tullberg M, Wikkelsø C, Starck G. Pre-and postoperative cerebral blood flow changes in patients with idiopathic normal pressure hydrocephalus measured by computed tomography (CT)perfusion. J Cereb Blood Flow Metab (2016) 36:1755-66. doi:10.1177/02716 78X15608521

32. Virhammar J, Laurell K, Ahlgren A, Cesarini KG, Larsson EM. Idiopathic normal pressure hydrocephalus: cerebral perfusion measured with pCASL before and repeatedly after CSF removal. J Cereb Blood Flow Metab (2014) 34:1771-8. doi: $10.1038 /$ jcbfm.2014.138

33. Ivkovic M, Reiss-Zimmermann M, Katzen H, Preuss M, Kovanlikaya I, Heier L, et al. MRI assessment of the effects of acetazolamide and external lumbar drainage in idiopathic normal pressure hydrocephalus. Fluids Barriers CNS (2015) 12:9. doi:10.1186/s12987-015-0004-z

34. Driver CJ, Rusbridge C, Cross HR, McGonnell I, Volk HA. Relationship of brain parenchyma within the caudal cranial fossa and ventricle size to syringomyelia in cavalier King Charles spaniels. J Small Anim Pract (2010) 51:382-6. doi:10.1111/j.1748-5827.2010.00952.x

35. Rusbridge C, Greitz D, Iskandar BJ. Syringomyelia: current concepts in pathogenesis, diagnosis and treatment. J Vet Intern Med (2006) 20:469-79. doi:10.1 111/j.1939-1676.2006.tb02884.x

36. Chen BC, Huang SC, Germano G, Kuhle W, Hawkins RA, Buxton D, et al. Noninvasive quantification of hepatic arterial blood flow with nitrogen-13-ammonia and dynamic positron emission tomography. J Nucl Med (1991) 32:2199-206.

37. Carroll TJ, Teneggi V, Jobin M, Squassante L, Treyer V, Hany TF, et al. Absolute quantification of cerebral blood flow with magnetic resonance, reproducibility of the method, and comparison with $\mathrm{H} 215 \mathrm{O}$ positron emision tomography. J Cereb Blood Flow Metab (2002) 22:1149-56. doi:10.1097/ 00004647-200209000-00013

38. Lin W, Celik A, Derdeyn C. Quantitative measurements of cerebral blood flow in patients with unilateral carotid artery occlusion: a PET and MR study. J Magn Res Imag (2001) 14:659-67. doi:10.1002/jmri.10021

39. Gobbel GT, Cann CE, Iwamoto HS, Fike JR. Measurement of regional cerebral blood flow in the dog using ultrafast computed tomography. Experimental validation. Stroke (1991) 22:772-9. doi:10.1161/ 01.STR.22.6.772

40. Peterson KL, MacLeod AG, Wisner ER, Larson RF, Pollard RE. Quantitative assessment of blood volume, blood flow, and permeability of the brain of clinically normal dogs by use of dynamic contrast-enhanced computed tomography. Am J Vet Res (2008) 69:45-50. doi:10.2460/ajvr.69.1.45

41. Hansen TD, Warner DS, Todd MM, Vust LJ, Trawick DC. Distribution of cerebral blood flow during halothane versus isoflurane anesthesia in rats. J Am Soc Anesthesiol (1988) 69:332-7. doi:10.1097/00000542-198809000-00008

42. Farber NE, Harkin CP, Niedfeldt J, Hudetz AG, Kampine JP, Schmeling WT. Region-specific and agent-specific dilation of intracerebral microvessels by volatile anesthetics in rat brain slices. Anesthesiology (1997) 87:1191-8. doi:10.1097/00000542-199711000-00024 
43. Matta BF, Heath KJ, Tipping K, Summors C. Direct cerebral vasodilatory effects of sevoflurane and isoflurane. Anesthesiology (1999) 91:677-80. doi:10.1097/00000542-199909000-00019

44. Wintermark M, Thiran JP, Maeder P, Schnyder P, Meuli R. Simultaneous measurement of regional cerebral blood flow by perfusion CT and stable xenon CT: a validation study. AJNR Am J Neuroradiol (2001) 22:905-14.

45. Essig M, Shiroishi MS, Nguyen TB, Saake M, Provenzale JM, Enterline D, et al. Perfusion MRI: the five most frequently asked technical questions. AJR Am J Roentgenol (2013) 200:24-34. doi:10.2214/AJR.12.9543

46. Hakim S, Adams RD. The special clinical problem of symptomatic hydrocephalus with normal cerebrospinal fluid pressure. Observations on cerebrospinal fluid hydrodynamics. J Neurol Sci (1965) 2:307-27. doi:10.1016/0022-510X(65)90016-X

47. Rekate HL. A consensus on the classification of hydrocephalus: its utility in the assessment of abnormalities of cerebrospinal fluid dynamics. Childs Nerv Syst (2011) 27:1535-41. doi:10.1007/s00381-011-1558-y

48. Momjian S, Owler BK, Czosnyka Z, Czosnyka M, Pena A, Pickard JD. Pattern of white matter regional cerebral blood flow and autoregulation in normal pressure hydrocephalus. Brain (2004) 127:965-72. doi:10.1093/ brain/awh131

49. Jeppsson A, Zetterberg H, Blennow K, Wikkelsø C. Idiopathic normal-pressure hydrocephalus: pathophysiology and diagnosis by CSF biomarkers. Neurology (2013) 80:1385-92. doi:10.1212/WNL.0b013e31828c2fda

50. Owler BK, Momjian S, Czosnyka Z, Czosnyka M, Pena A, Harris NG, et al. Normal pressure hydrocephalus and cerebral blood flow: a PET study of baseline values. J Cereb Blood Flow Metab (2004) 24:17-23. doi:10.1097/01. WCB.0000093326.88757.49

51. Owler BK, Pickard JD. Normal pressure hydrocephalus and cerebral blood flow: a review. Acta Neurol Scand (2004) 104:325-42. doi:10.1034/j. 1600-0404.2001.00092.x

52. Kristensen B, Malm J, Fagerland M, Hietala SO, Johansson B, Ekstedt J, et al. Regional cerebral blood flow, white matter abnormalities, and cerebrospinal fluid hydrodynamics in patients with idiopathic adult hydrocephalus syndrome. J Neurol Neurosurg Psychiatry (1996) 60:282-8. doi:10.1136/ jnnp.60.3.282
53. Loughin CA, Marino DJ. Atlantooccipital overlap and other craniocervical junction abnormalities in dogs. Vet Clin North Am Small Anim Pract (2016) 46:243-51. doi:10.1016/j.cvsm.2015.10.008

54. Scrivani PV, Freer SR, Dewey CW, Cerda-Gonzalez S. Cerebrospinal fluid signal-void sign in dogs. Vet Radiol Ultrasound (2009) 50:269-75. doi:10.1111/j.1740-8261.2009.01532.x

55. Schmidt MJ, Amort KH, Failing K, Klingler M, Kramer M, Ondreka N. Comparison of the endocranial- and brain volumes in brachycephalic dogs, mesaticephalic dogs and Cavalier King Charles spaniels in relation to their body weight. Acta Vet Scand (2014) 13:30. doi:10.1186/ 1751-0147-56-30

56. Schmidt MJ, Ondreka N, Sauerbrey M, Volk HA, Rummel C, Kramer M. Volume reduction of the jugular foramina in Cavalier King Charles Spaniels with syringomyelia. BMC Vet Res (2012) 6:158. doi:10.1186/1746-6148-8-158

57. Lu D, Lamb CR, Pfeiffer DU, Targett MF. Neurological signs and results of magnetic resonance imaging in 40 cavalier King Charles spaniels with Chiari type 1-like malformations. Vet Rec (2003) 153:60-3. doi:10.1136/ vr.153.9.260

58. Keong NC, Pena A, Price SJ, Czosnyka M, Czosnyka Z, Pickard JD. Imaging normal pressure hydrocephalus: theories, techniques, and challenges. Neurosurg Focus (2016) 41:E11. doi:10.3171/2016.7.FOCUS16194

59. Chrysikopoulos H. Idiopathic normal pressure hydrocephalus: thoughts on etiology and pathophysiology. Med Hypotheses (2009) 73:718-24. doi:10.1016/j.mehy.2009.04.044

Conflict of Interest Statement: The authors declare that the research was conducted in the absence of any commercial or financial relationships that could be construed as a potential conflict of interest.

Copyright (C) 2017 Schmidt, Kolecka, Kirberger and Hartmann. This is an open-access article distributed under the terms of the Creative Commons Attribution License (CC BY). The use, distribution or reproduction in other forums is permitted, provided the original author(s) or licensor are credited and that the original publication in this journal is cited, in accordance with accepted academic practice. No use, distribution or reproduction is permitted which does not comply with these terms. 